\title{
Association of UGT1A6 gene polymorphism with clinical outcome in pediatric epileptic patients on sodium valproate monotherapy
}

\author{
N. Banawalikar ${ }^{1 \oplus}$, S. Adiga ${ }^{2 \oplus 凶}$, U. Adiga $^{3 \oplus}$, V. Shenoy ${ }^{4 \oplus}$, S. Kumari ${ }^{3 \oplus}$, P. Shetty ${ }^{1 \oplus}$ \\ S. Shetty ${ }^{10}$, and K.P. Sharmila ${ }^{10}$ \\ ${ }^{1}$ Central Research Laboratory, KS Hegde Medical Academy, Mangalore, Karnataka, India \\ ${ }^{2}$ Department of Pharmacology, KS Hegde Medical Academy, Mangalore, Karnataka, India \\ ${ }^{3}$ Department of Biochemistry, KS Hegde Medical Academy, Mangalore, Karnataka, India \\ ${ }^{4}$ Department of Pediatrics, KS Hegde Medical Academy, Mangalore, Karnataka, India
}

\begin{abstract}
Pediatric epilepsy comprises chronic neurological disorders characterized by recurrent seizures. Sodium valproate is one of the common antiseizure medications used for treatment. Glucuronide conjugation is the major metabolic pathway of sodium valproate, carried out by the enzyme uridine $5^{\prime}$-diphosphate (UDP) glucuronosyl transferase (UGT) whose gene polymorphisms may alter the clinical outcome. The objective of this study was to assess the association between UGT1A6 genetic polymorphism and clinical outcome in terms of efficacy and tolerability in pediatric epileptic patients on sodium valproate monotherapy. Pediatric epileptic patients $(n=65)$ aged $2-18$ years receiving sodium valproate monotherapy for the past one month were included. Genetic polymorphism patterns of UGT1A6 (T19G, A541G, A552C) were evaluated by PCR-RFLP. Clinical outcome was seizure control during the 6 months observation period. Tolerability was measured by estimating the hepatic, renal, and other lab parameters. Out of 65 patients, TT (40\%), TG (57\%), and GG (3\%) patterns were observed in UGT1A6 (T19G) gene, AA (51\%), AG (40\%), and GG (9\%) in (A541G) gene, and AA (43\%), AC (43\%), and CC (14\%) in (A552C) gene. No statistical difference in clinical outcome was found for different UGT1A6 genetic polymorphism patterns. We concluded that different patterns of UGT1A6 genetic polymorphism were not associated with the clinical outcome of sodium valproate in terms of efficacy and tolerability. Sodium valproate was well-tolerated among pediatric patients with epilepsy and can be used as an effective antiseizure medication.
\end{abstract}

Key words: UGT1A6; Genetic polymorphism; Sodium valproate; Clinical outcome

\section{Introduction}

Epilepsy is a neurological disorder comprising heterogeneous and complex brain disorders of many seizure types and epilepsy syndromes $(1,2)$. The overall prevalence of epilepsy in India is estimated to be 3-11.9 per 1000 people (3). It is common practice for pediatricians and neurologists to begin long-term, daily antiseizure medications after a patient has experienced unprovoked seizures (4). Anti-epileptic drugs (AEDs) lead to satisfactory seizure control for about $60-70 \%$ of newly treated patients (5), while the remaining $30 \%$ of patients will have uncontrolled epilepsy with AED treatment for a prolonged period with recurrent seizures, adverse effects, and significantly increased risk of mortality and morbidity $(1,5)$. The goal of epilepsy treatment in the pediatric patient is to prevent the seizure attack for two years with an appropriate antiseizure medication with minimal adverse effects so that the drug can be slowly tapered off later.

Pharmacogenetics refers to the science about the genetic variations affecting drug metabolism, drug targets, or disease pathways leading to a varying response to the drug concerning its efficacy or adverse effects. Hence, it is important to investigate the role of genetic polymorphism that can alter drug metabolism and the effect of antiseizure medications.

Sodium valproate is a widely used broad-spectrum antiseizure medication due to its effect on different types of epilepsy. Glucuronidation and $\beta$-oxidation are the prominent metabolic pathways, while CYP-mediated oxidation is a minor pathway in the metabolization of sodium valproate drugs. Glucuronidation is catalyzed by uridine-5'diphosphate glucuronosyl transferases (UGTs), 
and polymorphisms affecting genes encoding for UGT enzymes may result in alterations of sodium valproate metabolism. Changes in the glucuronidation rate of sodium valproate may alter the blood level of the drug resulting in either insufficient plasma concentration that leads to reduced effectiveness of therapy or elevated plasma concentration of the drug that manifests as toxicity (6). Previous research has focused on UGT1A6 and CYP polymorphisms in the Chinese, Iranian, and Indian populations. Most of the studies have aimed at finding the pattern of genetic polymorphism and its effect on the serum concentration of the patient. However, currently available information on sodium valproate on UGT1A6 polymorphism and its association with clinical outcome in the Indian population is very limited.

The objectives of this study were to evaluate the pattern of UGT1A6 gene polymorphism in pediatric epileptic patients and assess the association of UGT1A6 gene polymorphism with clinical outcome in terms of efficacy and tolerability in pediatric epileptic patients on sodium valproate monotherapy.

\section{Material and Methods}

This cohort study was carried out at the pediatric department of Justice KS Hegde Charitable Hospital and Central Research Laboratory of KS Hegde Medical Academy, Mangalore, India, from February 2018 to December 2019. Sixty-five pediatric epileptic patients aged 2-18 years of either sex treated with sodium valproate monotherapy were enrolled. The Ethics Committee approval was obtained before the study. Written informed consent was obtained before enrollment. Inclusion criteria were patients diagnosed clinically by EEG and who were on a steady stable dose of $20 \mathrm{mg} / \mathrm{kg}$ per day sodium valproate twice/thrice a day for the past one month. Patients with a history or evidence of hepatitis or impaired renal functions or who were already on treatment with any other antiseizure medications or drugs that induced or inhibited sodium valproate metabolism were excluded.

Four milliliters of whole blood was collected with aseptic precautions once the patient fulfilled the criteria and during a 6-month follow-up visit. The sample was separated into 2 parts: $2 \mathrm{~mL}$ of EDTA whole blood was stored at $-80^{\circ} \mathrm{C}$ for genetic polymorphism analysis and $2 \mathrm{~mL}$ of whole blood in a plain vial was centrifuged at $1968 \mathrm{~g}$ for $5 \mathrm{~min}$ at $25^{\circ} \mathrm{C}$ to obtain serum, and then stored at $-80^{\circ} \mathrm{C}$ to analyze biochemical parameters. Liver function tests (albumin, total protein, total and direct bilirubin, serum glutamic oxaloacetic transaminase (SGOT), serum glutamic pyruvic transaminase (SGPT), alkaline phosphatase $(A L P)$ ), renal function test (blood urea, serum creatinine), platelet count, and serum amylase were estimated at the time of enrollment and at 6 months.

\section{Genotyping}

Genomic DNA from whole blood was isolated by phenol-chloroform extraction and ethanol precipitation method. The UGT1A6 (T19G, A541G, A552C) polymorphisms were analyzed by the polymerase chain reactionrestriction fragment length polymorphism (PCR-RFLP) method $(7,8)$. Amplification was performed in an MJ-Mini Thermal cycler (BioRad, Japan). PCR was conducted with an initial denaturation enzyme activation step at $95^{\circ} \mathrm{C}$ for $5 \mathrm{~min}$, amplification step for 35 cycles at $95^{\circ} \mathrm{C}$ for $30 \mathrm{~s}$, an annealing temperature for $30 \mathrm{~s}$, treatment at $72^{\circ} \mathrm{C}$ for $30 \mathrm{~s}$, and a final extension step at $72^{\circ} \mathrm{C}$ for $5 \mathrm{~min}$. PCR product was digested with each restriction enzyme (Hha I, Nsil, Fnu4HI), respectively, for T19G, A541G, A552C (Figure 1). The amplified product of DNA samples and restriction fragments was separated on a $2 \%$ agarose gel with ethidium bromide. The genotype was assigned based on the results of the analysis of the digestion patterns.

Parents were instructed to maintain a diary to record seizure attacks if any, duration of the attack, and change

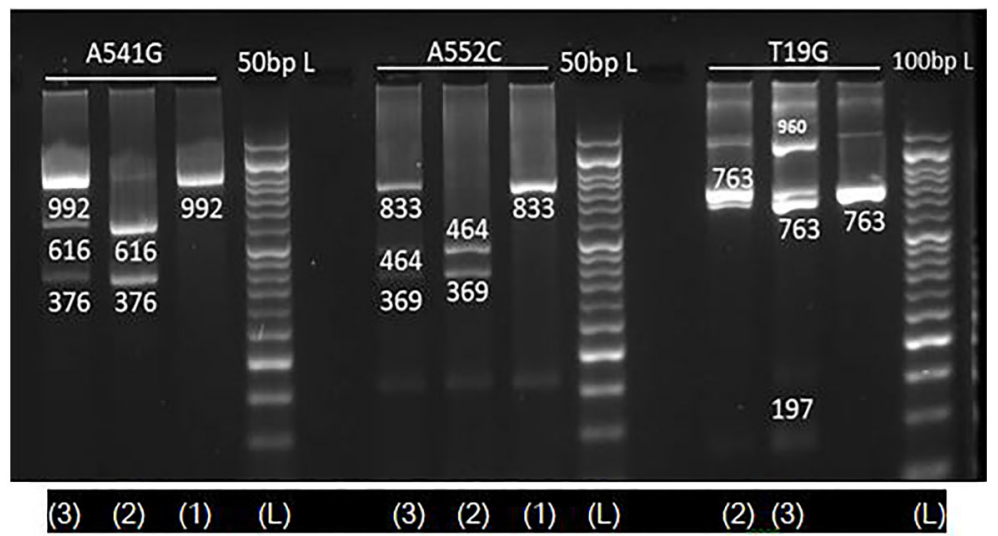

Figure 1. Electrophoresis gel image patterns of UGT1A6 (A541G, A552C, T19G) gene after digestion with Nsil, Fnu4HI, and Hhal, respectively. L: Ladder; lane (1): homozygosity for variant allele; lane (2): homozygosity for common allele; lane (3): heterozygosity. 
in the pattern from previous episodes, and the days the child had skipped the medication. They were also instructed to bring the child for a clinical visit in the event of nausea, vomiting, loss of appetite, jaundice, abdominal pain anytime or otherwise at the end of 6 months.

Clinical outcome was assessed in terms of efficacy and safety of sodium valproate. The drug efficacy was measured in terms of treatment responders or nonresponders. Responder to sodium valproate was defined as patients with no relapse or 1 episode of seizure attack in 6 consecutive months of follow-up and who tolerated sodium valproate monotherapy well without any adverse effect. Non-responders were those with two or more episodes of seizure attack in six months of follow-up or with any drug adverse effect after therapy initiation (9). Tolerability was assessed by comparing the biochemical parameters between baseline and at the six-month visit and by assessing whether patients experienced jaundice, pancreatitis, or signs of thrombocytopenia.

Table 1. Hardy-Weinberg equilibrium test for UGT1A6 gene.

\begin{tabular}{cccc}
\hline Genotype & Actual values & Expected values & P-value \\
\hline T19G & & & \\
TT & 26 & 30.5 & 6.58 \\
TG & 37 & 28.0 & \\
GG & 2 & 6.5 & \\
A541G & & 32.6 & 0.0716 \\
AA & 33 & 26.9 & \\
AG & 26 & 5.5 & \\
GG & 6 & & \\
A552C & & 27.1 & 0.2184 \\
AA & 28 & 29.7 & \\
AC & 28 & 8.2 & \\
CC & 9 &
\end{tabular}

The chi-squared test was used for statistical analysis.

\section{Sample size calculation}

Based on the genotype frequencies reported previously, considering a standard drug concentration of $23 \mu \mathrm{g} / \mathrm{mL}$ (10), and fixing the margin of error as 5 , the calculated sample size was 87 .

However, due to the change in treatment guidelines of pediatric epilepsy given by the Indian Pediatric Association (preference of levetiracetam over sodium valproate), we could enroll only 65 patients.

\section{Statistical analysis}

The collected data are reported as frequency and percentage for qualitative data and as means $\pm S D$ for quantitative data using SPSS software (version 16.0, IBM, USA). Comparison between responders and non-responders in different gene patterns of UGT1A6 studied was done by the chi-squared test. Biochemical parameters were compared between baseline and at 6 months follow-up by paired $t$-test in different age groups. A P-value $<0.05$ was considered statistically significant.

\section{Results}

The mean age of patients enrolled in the study was $7.38 \pm 3.67$ years. There were 34 male and 31 female patients and the mean body mass index was $16.89 \pm 4.56 \mathrm{~kg} / \mathrm{m}^{2}$.

Out of 65 patients, TT $(40 \%)$, TG $(57 \%)$, and GG $(3 \%)$ patterns were observed in UGT1A6 (T19G) gene, AA (51\%), AG (40\%), and GG (9\%) in (A541G) gene, and AA $(43 \%), A C(43 \%)$, and $C C(14 \%)$ in (A552C) gene. The frequencies were consistent with the Hardy-Weinberg equilibrium (Table 1). No statistical difference was found in clinical outcome with UGT1A6 (T19G, A541G, A552C) genetic polymorphisms (Table 2). There was a higher pattern of mutant alleles carriers for the genes UGT1A6 (T19G and A552C).

Table 2. Association of UGT1A6 gene polymorphisms with clinical outcome $(\mathrm{N}=65)$.

\begin{tabular}{|c|c|c|c|c|c|}
\hline \multirow[t]{2}{*}{ Genotype UGT1A6 } & \multirow[t]{2}{*}{ Polymorphism pattern } & \multirow[t]{2}{*}{ Polymorphism } & \multicolumn{2}{|c|}{ Clinical outcome } & \multirow[t]{2}{*}{ P-value } \\
\hline & & & $\begin{array}{c}\text { Responders } \\
(\mathrm{N}=53)(81.5 \%)\end{array}$ & $\begin{array}{l}\text { Non-responders } \\
(\mathrm{N}=12)(18.5 \%)\end{array}$ & \\
\hline \multirow[t]{3}{*}{ T19G } & TT & $40 \%$ & $20(37.8 \%)$ & $6(50 \%)$ & 0.318 \\
\hline & TG & $57 \%$ & $32(60.4 \%)$ & $5(41.6 \%)$ & \\
\hline & GG & $3 \%$ & $1(1.8 \%)$ & 1 (8.4\%) & \\
\hline \multirow[t]{3}{*}{ A541G } & AA & $51 \%$ & $28(52.8 \%)$ & $5(41.6 \%)$ & 0.568 \\
\hline & $A G$ & $40 \%$ & $21(39.6 \%)$ & $5(41.6 \%)$ & \\
\hline & GG & $9 \%$ & $4(7.6 \%)$ & $2(16.8 \%)$ & \\
\hline \multirow[t]{3}{*}{ A552C } & AA & $43 \%$ & $23(43.4 \%)$ & $5(41.6 \%)$ & 0.952 \\
\hline & $\mathrm{AC}$ & $43 \%$ & $23(43.4 \%)$ & $5(41.6 \%)$ & \\
\hline & $\mathrm{CC}$ & $14 \%$ & $7(13.2 \%)$ & $2(16.8 \%)$ & \\
\hline
\end{tabular}

Data are reported as $\mathrm{n}(\%)$. Chi-squared test. 
A total of 53 patients $(81.5 \%)$ responded well to the treatment without any relapse of seizure and 12 patients $(18.5 \%)$ had a relapse of seizure with two or more episodes. These patients were shifted to other antiseizure medications. No statistical difference was found in clinical efficacy (seizure control) with UGT1A6 (T19G, A541G, A552C) genetic polymorphism (Table 2). We have also analyzed the relative risk between the clinical outcome among carriers of wild and mutant allele types in UGT1A6 (T19G, A541G, and A552C). The wild-type gene pattern patients in $A 541 G$ seemed to have a better response in seizure control compared to mutant allele type carriers even though the P-value was not statistically significant. It was also observed that the wild-type pattern seemed to have a poor response in terms of efficacy at T19G and A552C loci (Table 3).

Fifty-two patients (80\%) were diagnosed with generalized tonic-clonic seizures (GTCS) and 13 patients (20\%) were diagnosed with focal seizures with awareness impairment (FSAI). There was no statistically significant difference in relative risk of poor clinical outcome among patients of GTCS and FSAl of different gene patterns (Table 4). However, GTCS patients with T19G and A552C of wild-type allele had a higher risk of having a poor response (poor seizure control) compared to FSAI patients.

Except for two patients, all children had normal liver, hematological, and renal function parameters at baseline and 6 months follow-up (Table 5). One patient developed acute pancreatitis, with elevated serum lipase and features suggestive of pancreatitis in ultrasound. De-challenge was given and the patient recovered completely and shifted to levetiracetam. Causality assessment was done using the Naranjo algorithm, which revealed a score of +6 suggesting the probable nature of the adverse drug effect was from sodium valproate.

\section{Safety parameters}

We divided the biochemical and hematological parameters into three subgroups as there may be variations by age group. A statistically significant difference was found in the values at 6-month assessment compared to baseline values in SGOT, SGPT, serum amylase, direct

Table 3. Relative risk of a poor clinical outcome in various gene polymorphisms.

\begin{tabular}{|c|c|c|c|c|c|c|}
\hline \multirow[t]{2}{*}{ Gene loci } & \multirow[t]{2}{*}{ Pattern } & \multirow[t]{2}{*}{ Probability of poor response } & \multicolumn{2}{|c|}{ Clinical outcome (N, \%) } & \multirow[t]{2}{*}{ Relative risk } & \multirow[t]{2}{*}{ P-value } \\
\hline & & & $\begin{array}{c}\text { Good } \\
(\mathrm{N}=53)(81.5 \%)\end{array}$ & $\begin{array}{c}\text { Poor } \\
(\mathrm{N}=12)(18.5 \%)\end{array}$ & & \\
\hline \multirow[t]{2}{*}{ T19G } & Wild & 0.23 & $20(37.8 \%)$ & $6(50 \%)$ & 1.53 & 0.52 \\
\hline & Mutant & 0.15 & $33(62.2 \%)$ & $6(50 \%)$ & & \\
\hline \multirow[t]{2}{*}{ A541G } & Wild & 0.15 & $28(52.8 \%)$ & $5(41.6 \%)$ & 0.71 & 0.54 \\
\hline & Mutant & 0.21 & $25(47.2 \%)$ & $7(58.4 \%)$ & & \\
\hline \multirow[t]{2}{*}{ A552C } & Wild & 0.17 & $23(43.4 \%)$ & $5(41.6 \%)$ & 1.01 & $>0.99$ \\
\hline & Mutant & 0.18 & $30(56.4 \%)$ & $7(58.4 \%)$ & & \\
\hline
\end{tabular}

Data are reported as $\mathrm{n}(\%)$. Fisher's exact test.

Table 4. Relative risk of poor clinical outcome in various types of seizures.

\begin{tabular}{|c|c|c|c|c|c|c|}
\hline \multirow[t]{2}{*}{ Gene loci } & \multirow[t]{2}{*}{ Seizure type } & \multirow[t]{2}{*}{ Gene pattern } & \multicolumn{2}{|c|}{ Clinical outcome } & \multirow[t]{2}{*}{ Relative risk } & \multirow[t]{2}{*}{ P-value } \\
\hline & & & Good N=52 $(80 \%)$ & Poor $\mathrm{N}=13(20 \%)$ & & \\
\hline \multirow[t]{4}{*}{ T19G } & GTCS & Wild & $11(21.2 \%)$ & $5(38.5 \%)$ & 2.25 & 0.25 \\
\hline & & Mutant & $30(57.7 \%)$ & $6(46.1 \%)$ & & \\
\hline & FSAI & Wild & $9(17.3 \%)$ & $1(7.7 \%)$ & 0.42 & 0.30 \\
\hline & & Mutant & $2(3.8 \%)$ & $1(7.7 \%)$ & & \\
\hline \multirow[t]{4}{*}{ A541G } & GTCS & Wild & $22(42.4 \%)$ & $5(38.5 \%)$ & 0.92 & $>0.99$ \\
\hline & & Mutant & $19(36.5 \%)$ & $6(46.1 \%)$ & & \\
\hline & FSAI & Wild & $6(11.5 \%)$ & 0 & 0 & 0.46 \\
\hline & & Mutant & $5(9.6 \%)$ & $2(15.4 \%)$ & & \\
\hline \multirow[t]{4}{*}{ A552C } & GTCS & Wild & $16(30.8 \%)$ & $5(38.5 \%)$ & 1.47 & 0.50 \\
\hline & & Mutant & $25(48.1 \%)$ & $6(46.1 \%)$ & & \\
\hline & FSAI & Wild & $7(13.5 \%)$ & 0 & 0 & 0.19 \\
\hline & & Mutant & $4(7.6 \%)$ & $2(15.4 \%)$ & & \\
\hline
\end{tabular}

Data are reported as $n$ (\%). Fisher's exact test. GTCS: generalized tonic-clonic seizures; FSAI: focal seizures with awareness impairment. 
Table 5. Biochemical parameters between baseline and 6 months in the different age groups.

\begin{tabular}{|c|c|c|c|c|c|c|c|c|c|}
\hline \multirow[t]{2}{*}{ Parameters } & \multicolumn{3}{|c|}{2 years } & \multicolumn{3}{|c|}{$6-12$ years } & \multicolumn{3}{|c|}{$12-18$ years } \\
\hline & Basal & 6 months & P-value & Basal & 6 months & $\mathrm{P}$-value & Basal & 6 months & P-value \\
\hline Albumin (g/dL) & $4.3 \pm 0.33$ & $4.2 \pm 0.33$ & 0.674 & $4.5 \pm 0.39$ & $4.4 \pm 0.26$ & 0.363 & $4.6 \pm 0.28$ & $4.6 \pm 0.24$ & 0.984 \\
\hline Total protein (g/dL) & $6.8 \pm 0.37$ & $7.2 \pm 0.60$ & 0.117 & $7.4 \pm 0.74$ & $7.4 \pm 0.5$ & 0.974 & $7.5 \pm 0.49$ & $7.7 \pm 0.3$ & 0.745 \\
\hline SGOT (IU/L) & $33.8 \pm 10$ & $28.3 \pm 7.8$ & 0.215 & $27.2 \pm 7$ & $26.9 \pm 9.5$ & $0.04^{*}$ & $26.7 \pm 15$ & $23.2 \pm 5$ & 0.326 \\
\hline SGPT (IU/L) & $14.7 \pm 6$ & $14.5 \pm 7.2$ & 0.091 & $11.6 \pm 4$ & $12 \pm 4.8$ & $0.009^{*}$ & $16.3 \pm 16$ & $13.1 \pm 3.6$ & 0.173 \\
\hline ALP (IU/L) & $219 \pm 93$ & $186 \pm 49$ & 0.881 & $198 \pm 53$ & $217 \pm 76$ & 0.267 & $177 \pm 84$ & $158 \pm 78$ & 0.578 \\
\hline Bil Dir (mg/dL) & $0.07 \pm 0.03$ & $0.09 \pm 0.04$ & 0.565 & $0.16 \pm 0.1$ & $0.11 \pm 0.05$ & $0.034^{\star}$ & $0.14 \pm 0.13$ & $0.12 \pm 0.08$ & $0.028^{*}$ \\
\hline Bil Tot (mg/dL) & $0.2 \pm 0.1$ & $0.2 \pm 0.1$ & 0.496 & $0.34 \pm 0.3$ & $0.32 \pm 0.14$ & $0.002^{*}$ & $0.38 \pm 0.2$ & $0.32 \pm 0.2$ & $0.032^{*}$ \\
\hline Urea (mg/dL) & $23.6 \pm 9.4$ & $19.2 \pm 9.1$ & 0.928 & $21.3 \pm 6.5$ & $21.3 \pm 7.4$ & 0.850 & $20.1 \pm 7.7$ & $20.1 \pm 7.2$ & 0.915 \\
\hline Creat (mg/dL) & $0.40 \pm 0.2$ & $0.35 \pm 0.2$ & 0.242 & $0.53 \pm 0.2$ & $0.36 \pm 0.1$ & $0.017^{*}$ & $0.64 \pm 0.2$ & $0.53 \pm 0.1$ & 0.119 \\
\hline Amylase (IU/L) & $84.4 \pm 32$ & $73.3 \pm 29$ & 0.301 & $93.3 \pm 54$ & $84.8 \pm 27$ & $0.012^{*}$ & $87.3 \pm 36$ & $90.3 \pm 44$ & $0.038^{*}$ \\
\hline Platelet (Lk/cum) & $2.9 \pm 0.33$ & $2.7 \pm 0.47$ & 0.167 & $2.8 \pm 0.78$ & $2.5 \pm 0.45$ & 0.342 & $2.8 \pm 0.32$ & $2.7 \pm 0.35$ & 0.092 \\
\hline
\end{tabular}

Data are reported as means \pm SD. SGOT: serum glutamic oxaloacetic transaminase; SGPT: serum glutamic pyruvic transaminase; ALP: alkaline phosphatase; Bil Dir: direct bilirubin; Bil Tot: total bilirubin. ${ }^{*} \mathrm{P} \leqslant 0.05$ (paired $t$-test). Bold type indicates statistical significance.

Table 6. Prevalence of UGT1A6 gene polymorphisms in children with epilepsy of various populations based on published studies.

\begin{tabular}{|c|c|c|c|c|c|c|c|c|}
\hline \multirow{2}{*}{$\begin{array}{l}\text { Genotype } \\
\text { UGT1A6 }\end{array}$} & \multicolumn{4}{|c|}{ Chinese } & \multirow{2}{*}{$\begin{array}{c}\text { Japanese } \\
\begin{array}{c}\text { Saeki et al. (14) } \\
(n=195)\end{array}\end{array}$} & \multirow{2}{*}{$\begin{array}{c}\text { Caucasian } \\
\begin{array}{c}\text { Lampe et al. (15) } \\
(n=100)\end{array}\end{array}$} & \multirow{2}{*}{ 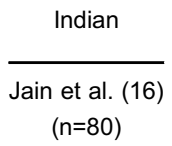 } & \multirow{2}{*}{$\begin{array}{c}\text { Indian } \\
\begin{array}{c}\text { Current study } \\
(n=65)\end{array}\end{array}$} \\
\hline & $\begin{array}{l}\text { Hung et al. (10) } \\
\qquad(n=162)\end{array}$ & $\begin{array}{l}\text { Guo et al. (11) } \\
\qquad(n=98)\end{array}$ & $\begin{array}{l}\text { Chu et al. }(12) \\
\quad(n=242)\end{array}$ & $\begin{array}{l}\text { Xing et al. (13) } \\
\quad(n=534)\end{array}$ & & & & \\
\hline \multicolumn{9}{|l|}{ T19G } \\
\hline TT & $62.4 \%$ & $54.3 \%$ & NS & NS & NS & NS & $45 \%$ & $40 \%$ \\
\hline TG & $30.9 \%$ & $39.4 \%$ & NS & NS & NS & NS & $39 \%$ & $57 \%$ \\
\hline GG & $6.8 \%$ & $6.4 \%$ & NS & NS & NS & NS & $16 \%$ & $3 \%$ \\
\hline \multicolumn{9}{|l|}{ A541G } \\
\hline $\mathrm{AA}$ & $64.8 \%$ & $56.4 \%$ & $65 \%$ & $60 \%$ & $62 \%$ & $46 \%$ & $49 \%$ & $51 \%$ \\
\hline$A G$ & $29.6 \%$ & $39.4 \%$ & $33 \%$ & $35 \%$ & $33 \%$ & $43 \%$ & $39 \%$ & $40 \%$ \\
\hline GG & $5.6 \%$ & $4.3 \%$ & $2 \%$ & $5 \%$ & $5 \%$ & $11 \%$ & $12 \%$ & $9 \%$ \\
\hline \multicolumn{9}{|l|}{ A552C } \\
\hline $\mathrm{AA}$ & $62.9 \%$ & $55.3 \%$ & $57 \%$ & $57 \%$ & NS & $48 \%$ & $44 \%$ & $43 \%$ \\
\hline$A C$ & $30.3 \%$ & $39.4 \%$ & $39 \%$ & $37 \%$ & NS & $42 \%$ & $40 \%$ & $43 \%$ \\
\hline $\mathrm{CC}$ & $6.8 \%$ & $4.3 \%$ & $4 \%$ & $6 \%$ & NS & $10 \%$ & $16 \%$ & $14 \%$ \\
\hline
\end{tabular}

NS: not studied.

bilirubin, total bilirubin, and serum creatinine in the age group of 6-12 years. In the age group of >12-18 years, direct bilirubin, total bilirubin, and serum amylase values were statistically different between baseline and 6 months. Except for one patient who developed acute pancreatitis, all the remaining patients had no adverse effect. Three patients had elevated SGOT and two had elevated ALP levels, however, only one patient had severe abdominal pain, later found to have acute pancreatitis.

\section{Discussion}

Many researchers have investigated the role of gene polymorphisms in the pharmacokinetics and pharmacodynamics aspects of sodium valproate. The pattern of
UGT1A6 gene polymorphism was slightly different in the Indian population compared to the Chinese population concerning UGT1A6 (T19G, A541G, A552C type) (Table 6) (10-17). In the Chinese pediatric epileptic population, the predominant genetic polymorphism pattern observed was wild type while in our study population the predominant pattern observed was mutant type. However, there was no difference in the genetic pattern between our patients (South Indian) and North Indian pediatric epileptic patients $(8,16)$.

Different studies have reported different observations as to UGT gene polymorphism and its effect on sodium valproate concentration. Jain et al. (16), Wang et al. (18), and Chu et al. (12) reported no significant effect of polymorphisms on serum valproate concentrations. None 
of the available literature to date has explained the influence of UGT genetic variants on the clinical outcome of sodium valproate monotherapy. However, Hung et al. (10) reported that patients showing good drug response (seizure-free/good seizure control) should be evaluated for multiple genetic influences including UGT1A6 gene polymorphism on sodium valproate to guide dosing, seizure control, and adverse drug reactions. The authors concluded that carriers of the variant UGT1A6 19T $>G$, $541 \mathrm{~A}>\mathrm{G}$, and $552 \mathrm{~A}>\mathrm{C}$ alleles tend to require higher sodium valproate dosages and lower concentration-todose ratios $(C D R s)$ than non-carriers $(P<0.0001)$ and the homozygous carriers also seemed to require higher sodium valproate dosages and lower CDRs $(P<0.0001)$.

Munisamy et al. (7) reported that UGT1A6 552A $>$ C polymorphism plays a significant role in the elimination half-life, which was longer, and the clearance rate was lower in poor metabolizers at a steady-state concentration of sodium valproate. This showed toxicity in the intermediate metabolizers group or the extensive metabolizers group and it, thereby, has an impact on the toxicity of the sodium valproate drug found in the North Indian epileptic population. Jain et al. (16) reported that there was no significant association between sodium valproate doses, serum valproate concentration, and UGT1A6 genotypes in the North Indian population of children with epilepsy. Algharably et al. (19) reported in an Egyptian study that gene polymorphism of UGT1A6 at 541A $>$ G and 552A $>C$ loci may influence the clinical response profile of pediatric epileptic patients including both adverse drug reactions

\section{References}

1. Tejada J, Costa KM, Bertti P, Garcia-Cairasco N. The epilepsies: complex challenges needing complex solutions. Epilepsy Behav 2013; 26: 212-228, doi: 10.1016/j.yebeh. 2012.09.029.

2. Berg AT, Berkovic SF, Brodie MJ, Buchhalter J, Cross JH, van Emde Boas W, et al. Revised terminology and concepts for organization of seizures and epilepsies: report of the ILAE Commission on Classification and Terminology, 20052009. Epilepsia 2010; 51:676-685, doi: 10.1111/j.15281167.2010.02522.x.

3. Amudhan S, Gururaj G, Satishchandra P. Epilepsy in India I: epidemiology and public health. Ann Indian Acad Neurol 2015; 18: 263-277, doi: 10.4103/0972-2327.160093.

4. Cockerell OC, Johnson AL, Sander JW, Shorvon SD. Prognosis of epilepsy: a review and further analysis of the first nine years of the British National General Practice Study of Epilepsy, a prospective population-based study. Epilepsia 1997; 38: 31-46, doi: 10.1111/j.1528-1157.1997.tb01075.x.

5. Sillanpaa M, Schmidt D. Natural history of treated childhoodonset epilepsy: prospective, long-term population-based study. Brain 2006; 129: 617-624, doi: 10.1093/brain/awh726.

6. Chatzistefanidis D, Georgiou I, Kyritsis AP, Markoula S. Functional impact and prevalence of polymorphisms involved in the hepatic glucuronidation of valproic acid. and seizure activity. According to our study, the nonresponders of $\mathrm{A} 541 \mathrm{G}$ wild type was $15.16 \%$ and the mutant, it was $21.82 \%$ at the end of 6 months. A study conducted in the Egyptian pediatric population showed $47 \%$ non-response in wild-type and $52.9 \%$ in mutant-type carriers. The non-responder rate was $17.79 \%$ in patients with wild-type patterns in the A552C loci and $18.92 \%$ in patients with mutant type. A study done in the Egyptian pediatric population showed $55.9 \%$ non-response in wildtype and $44.1 \%$ in mutant-type carriers. However, in our study, we could not find any association between the different patterns of polymorphism with clinical efficacy and tolerability.

The limitation of our study was the small sample size. In the present context of the changed protocol in the treatment of pediatric epilepsy (preference of levetiracetam over sodium valproate) only metacentric studies involving different regions may answer our limitation and add quality to the data in terms of genetic pattern.

We conclude that different patterns of UGT1A6 genetic polymorphism were not associated with the clinical outcome of sodium valproate in terms of efficacy and tolerability in epileptic pediatric patients. Sodium valproate was well tolerated and can be used as an effective antiseizure medication in this population.

\section{Acknowledgment}

We thank NITTE (Deemed to be University) for funding this study.

Pharmacogenomics 2012; 13: 1055-1071, doi: 10.2217/ pgs.12.78.

7. Munisamy M, Tripathy M, Behari M, Raghavan S, Jain DC, Ramanujam B, et al. The effect of uridine disphosphate glucuronosyltransferase (UGT) 1A6 genetic polymorphism on valproic acid pharmacokinetics in indian patients with epilepsy: a pharmacogenetic approach. Mol Diagn Ther 2013; 17: 319-326, doi: 10.1007/s40291-013-0041-8.

8. Ciotti M, Marrone A, Potter C, Owens IS. Genetic polymorphism in the human UGT1A6 (planar phenol) UDP-glucuronosyl transferase: pharmacological implications. Pharmacogenetics 1997; 7: 485-495, doi: 10.1097/00008571-199712 000-00007.

9. Hans Carpay, William Arts. Outcome assessment in epilepsy: available rating scales for adults and methodological issues pertaining to the development of scales for childhood epilepsy. Epilepsy Res 1996; 24: 127-136, doi: 10.1016/0920-1211(96)00013-7.

10. Hung CC, Ho JL, Chang WL, Tai JJ, Hsieh TJ, Hsieh YW, et al. Association of genetic variants in six candidate genes with valproic acid therapy optimization. Pharmacogenomics 2011; 12: 1107-1117, doi: 10.2217/pgs.11.64.

11. Guo $\mathrm{Y}$, Cheng $\mathrm{Hu}$, Xiaojing He, FengQiu, Limei Zhao. Effects of UGT1A6, UGT2B7, and CYP2C9 genotypes on 
plasma concentrations of valproic acid in Chinese children with epilepsy. Drug Metab and Pharmacokinetics 2012; 27: 536-542, doi: 10.2133/dmpk.DMPK-11-NT-144.

12. Chu XM, Zhang LF, Wang GJ, Zhang SN, Zhou JH, Hao HP. Influence of UDP-glucuronosyltransferase polymorphisms on valproic acid pharmacokinetics in Chinese epilepsy patients. Eur J Clin Pharmacol 2012; 68: 1395-1401, doi: 10.1007/s00228-012-1277-7.

13. Xing $Y$, Yang L, Wang L, Shao L, Wei Z, Xuan J, et al. Systematic screening for polymorphisms within the UGT1A6 gene in three Chinese populations and function prediction through structural modeling. Pharmacogenomics 2009; 10 : 741-752, doi: 10.2217/pgs.09.21.

14. Saeki M, Saito Y, Jinno H, Sai K, Kaniwa N, Ozawa S, et al. Genetic polymorphisms of UGT1A6 in a Japanese population. Drug Metab Pharmacokinet 2005; 20: 85-90, doi: 10.2133/dmpk.20.85.

15. Lampe JW, Bigler J, Horner NK, Potter JD. UDP-glucuronosyl transferase (UGT1A $1{ }^{*} 28$ and UGT1A6*2) polymorphisms in Caucasians and Asians: relationships to serum bilirubin concentrations. Pharmacogenetics 1999; 9: 341-349, doi: 10.1097/00008571-199906000-00009.

16. Jain P, Shastri S, Gulati S, Kaleekal T, Kabra M, Gupta N, et al. Prevalence of UGT1A6 polymorphisms in children with epilepsy on valproate monotherapy. Neurol India 2015; 63: 35-39, doi: 10.4103/0028-3886.170098.

17. Nandith PB, Adiga U, Shenoy V, Adiga S. UGT1A6 and UGT2B7 gene polymorphism and its effect in pediatric epileptic patients on sodium valproate monotherapy. Indian J Pediatr 2021; 5: 1-7.

18. Wang Y, Gao L, Liu YP, Huang NN, Xu SJ and Ma DJ. Effect of UGTIA6 A541G genetic polymorphism on the metabolism of valproic acid in Han epileptic children from Henan [in Chinese]. Zhongguo Dang Dai Er Ke Za Zhi 2010; 12: 429-432.

19. Algharably EA, El Hamamsy M, Hassanein SM, Mohamed NA, Mahran MZ, EISawy MA, et al. The effect of UGT1A6 polymorphism at two loci on the clinical response to valproic acid in epileptic children. Int J Pharm Sci Res 2016; 7: 3986-3994. 